\title{
Bovine papillomavirus E5 and E7 oncoproteins in naturally occurring tumors: are two better than one?
}

\author{
Annunziata Corteggio, Gennaro Altamura, Franco Roperto and Giuseppe Borzacchiello*
}

\begin{abstract}
Bovine papillomaviruses (BPVs) are oncogenic DNA viruses, which mainly induce benign lesions of cutaneous and/ or mucosal epithelia in cattle. Thirteen (BPV 1-13) different viral genotypes have been characterized so far. BPVs are usually species-specific but BPV 1/2 may also infect equids as well as buffaloes and bison and cause tumors in these species. BPV-induced benign lesions usually regress, however occasionally they develop into cancer particularly in the presence of environmental carcinogenic co-factors. The major transforming protein of BPV is E5, a very short hydrophobic, transmembrane protein with many oncogenic activities. E5 contributes to cell transformation through the activation of the cellular $\beta$ receptor for the platelet-derived growth factor (PDGF $\beta-r$ ), it also decreases cell surface expression of major histocompatibility complex class I (MHCI) causing viral escape from immunosurveillance, and plays a role in the inhibition of the intracellular communication by means of aberrant connexin expression. E7 is considered as a weak transforming gene, it synergies with E5 in cell transformation during cancer development. E7 expression correlates in vivo with the over-expression of $\beta 1$-integrin, which plays a role in the regulation of keratinocytes proliferation and differentiation. Additionally, E7 is involved in cell-mediated immune responses leading to tumour rejection, in anoikis process by direct binding to p600, and in invasion process by upregulation of Matrix metalloproteinase1 (MMP-1) expression. Studies on the role of BPV E5 and E7 oncoproteins in naturally occurring tumours are of scientific value, as they may shed new light on the biological role of these two oncogenes in cell transformation.
\end{abstract}

Keywords: Bovine papillomavirus, Cow, E5, E7, Equids, Papillomas, Sarcoid, Viral oncogenes

\section{Introduction}

Papillomaviruses (PVs) are small oncogenic DNA viruses infecting humans as well as different animal species, causing benign hyperproliferative lesions of both mucosal and cutaneous epithelia or malignant cancers. BPVs belong to the PV family and infection of bovines by BPVs is associated with the development of cutaneous papillomas, alimentary and urinary bladder tumours. BPVs are usually species-specific, and, even in experimental conditions, do not infect any host other than the natural one. However, BPV type 1 (BPV-1) and BPV type 2 (BPV-2) represent a well known case of cross-species infection since they also infect equids as well as buffaloes and bisons [1-4]. BPV-induced benign lesions

\footnotetext{
* Correspondence: borzacch@unina.it

Department of Pathology and Animal Health, University of Naples Federico II, Via Veterinaria, Napoli 1 80137, Italy
}

usually regress, however occasionally they develop into cancer of both epithelial and mesenchymal origin. The major transforming protein of BPV is E5, a very small membrane-associated protein with potent biological activities [5]. The carcinogenic functions of E5 have been largely investigated in vitro, however many recent findings have highlighted the role of this oncoprotein also in naturally occurring oncogenesis.

BPV E7 is considered as a transforming gene although very little is known about E7-mediated mechanisms underlying carcinogenesis, so far. It has been hypothesized that BPV-E7 cooperates with E5 in cell transformation [6]. BPV has largely contributed as animal model to understand the genetics of PVs, the role of the early genes in cell transformation and still continues to do so due to very recent discoveries in naturally occurring equine and bovine tumours [7]. \\ C Biomed Central}


Furthermore, spontaneously arising BPV induced tumors are of interest not only in veterinary pathology but also from a comparative point of view. BPV E5 and E7 oncogenes have been studied at length up-to-date opening new scenarios about the possible role of these oncogenes in PVinduced cell transformation.

This review, will describe in brief the biological features of BPV E5 and E7 oncoproteins and will focus on the activity and the expression patterns of the two oncoproteins in vivo in naturally occurring bovine and equine tumours.

\section{BPV oncoproteins: general biological characteristics}

All PVs have a circular double-stranded DNA genome. The genetic organisation of BPVs is similar to that of other PVs. The open reading frames (ORFs) are divided into early and late regions. The early region encodes non structural proteins from E1 to E7. The late region encodes two structural proteins: L1 and L2. In addition, a non-coding long control region provides the cis-regulatory elements necessary for viral replication and transcription [8]. The transforming activities of BPVs are due to three early viral genes: E5, E6, and E7. The specific contribution of each oncogene in transforming process varies between different BPVs. Some BPVs (BPV-3,-4,-6) lack the E6 gene, although they are able to give a successful infection and to induce tumours [9].

Despite the clear evidence that E6 is an efficient transforming protein in vitro $[10,11]$, the activity of E6 in vivo in naturally occurring tumours still remains uncertain and needs to be investigated.

The growing body of recent studies in vivo highlights the central role of E5 and E7 expression/activity in tumour development strengthening the focus of this review.

\section{E5 oncogene}

The major transforming protein of BPV is E5, a very small, membrane associated protein with different biological activities and critical to the transformation process $[5,12,13]$. BPV-1 E5 is the shortest oncoprotein yet characterized being only 44-aminoacid long [12,14,15]. Genetic and biochemical studies have revealed that the E5 protein can be divided into two distinct domains: an amino-terminal domain (residues 1-32), consisting of strongly hydrophobic aminoacid residues and a single hydrophilic aminoacid, and an hydrophilic carboxyl-terminal domain (residues 33-44). The hydrophobic domain is presumed to mediate the association with cellular membranes and to function as a signal-anchor domain whereas the hydrophilic domain is believed to interact with important cellular regulatory proteins [16]. In accordance with its predicted hydrophobic composition, the E5 protein spontaneously localizes in the membranous compartment where it is present as a dimer.
The C-terminal domain is involved in E5 dimer and oligomer formation. Oligomerization appears to be essential for the transforming activity [12,17]. E5 accumulation in the late (trans-) Golgi apparatus (GA) is critical for its mitogenic signalling. Retention of E5 in the ER and early (cis-) Golgi causes loss of transformation activity [18]. Mutagenesis analysis underscore the critical role of individual aminoacid residues in mediating E5 biological activities. Several studies indicate that the hydrophobic nature of BPV E5 and a handful of important aminoacidic residues confer transforming activity [17]. These essential aminoacids are the most well conserved among related papillomaviruses [19].

E5 induces cell transformation through the activation and/or impairing of several intracellular targets involved in cell proliferation and survival [5].

\section{E7 oncogene}

The BPV-1 E7 oncogene is a zinc binding protein 127 aminoacids long. E7 does not show a strong independent transforming activity, but when it is coexpressed with E5, its transformation capacity is pronouncedly increased [6]. Mutants lacking the E7 open reading frame are still able to induce transformation but with lower efficiency [20]. It is not clear how BPV-1 E7 contributes to cell transformation as this oncoprotein, in contrast to HPV-E7, lacks the LXCXE motif that mediates the binding to the pRB family proteins [21]. It has been reported that the transformation activity of BPV-1 E7 is mediated, at least in part, by its ability to bind p600. The binding between BPV-1 E7 and p600 contributes to cell transformation by inhibiting the anoikis, a type of apoptosis, which is commonly encountered in cancer cells, allowing them to survive in the absence of normal cell-matrix interactions [22]. In addition, BPV-1 E7 seems to sensitize the cells to TNF-induced apoptosis [23,24]. On the contrary E7 is the major transforming protein of BPV-4 as defined in in vitro systems [25,26]. BPV-4 E7 contains two Cys-X-X-Cys motif and the pRb-binding domain, both are critical for its transforming activity [25].

Most of the studies on BPVs have been performed in in vitro systems where the biological characteristics and functions of BPV oncoproteins have been largely investigated, however the functions of BPV oncoproteins in vivo are less understood. Recent insights into E5 and E7 biological activities strenghten the pivotal role of these oncoproteins in cell transformation in naturally occurring tumours.

\section{BPV E5 and E7 in naturally occurring tumours}

BPV infection is associated to cutaneous papillomas, alimentary fibropapillomas and urinary bladder tumours in cattle and to sarcoids in equids. 


\section{Bovine cutaneous papillomas}

Cutaneous papillomatosis is characterized by the presence of multiple benign exophitic proliferations of the epithelia (Figure 1A). At least 11 different types of BPVs have been identified in association with cutaneous papillomas and fibropapillomas (BPV-1, BPV-2, BPV-5, BPV6, BPV-7, BPV-8, BPV-9, BPV-10, BPV-11, BPV-13) $[1,27,28]$. When BPV-infection of the skin causes also the transformation of dermal fibroblasts and epithelial

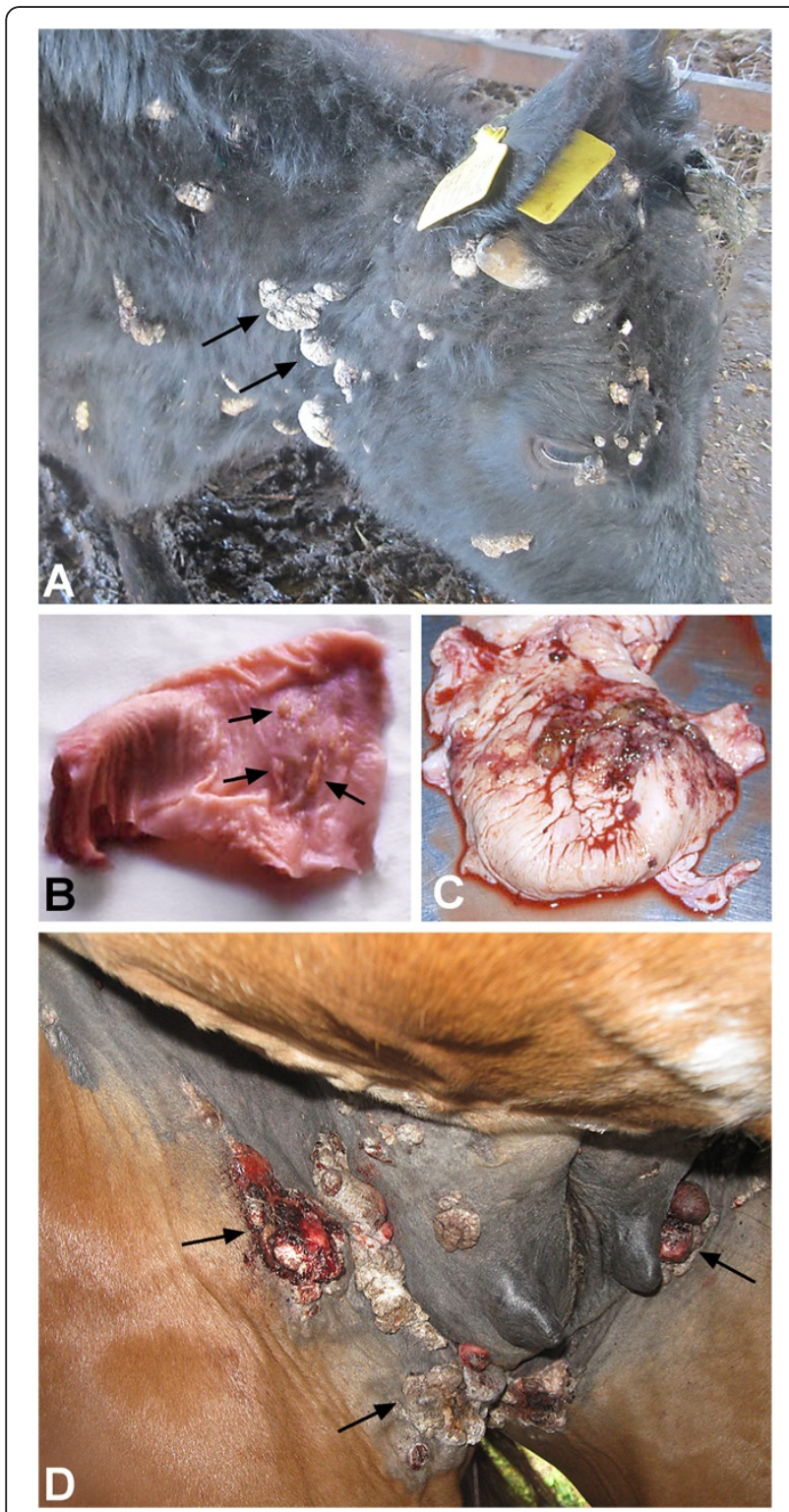

Figure 1 Examples of BPV induced tumours in its natural host, bovine, and in its heterologous host, horse. A. Persistent papillomatosis of the skin in a cow. B. Diffuse papillomatosis of the oesophagus in a cows, the arrows indicate the papillomas. $\mathbf{C}$. Multiple haemangiosarcomas and urothelial carcinoma of the urinary bladder in a cows. D. Multiple nodular sarcoids in the paragenital and mammary region in a horse. acanthosis, the lesion is defined as fibropapilloma. Papillomas and fibropapillomas may localize in different body sites in cattle [29].

During papilloma formation, the expression of BPV early and late genes is strictly regulated. The virus initially infects the basal keratinocytes. The early genes are expressed in undifferentiated basal and suprabasal layers, determining the re-entry of spinous cells into the cell cycle and amplification of viral DNAs in a process termed vegetative viral replication [4]. The distribution of BPV E5 and E7 proteins have been studied in bovine fibropapillomas.

In bovine fibropapillomas two sites of E5 expression have been identified within the epithelium: the cytoplasm of basal cells and superficial granular cell layer. High concentrations have been noted for E5 protein in the differentiated tumour keratinocytes. The granular staining pattern is associated with the sites of viral capsid synthesis [30]. The expression pattern of E5 and its localization in the lesions are suggestive for E5 actively contributing to successfull BPV (de novo) infection.

The localization in the basal layers indicates that E5 may have a role during the early stages of viral infection and tumour development and it may lead to sustained cell proliferation to favor virus-infected cells. Its presence in the more differentiated layers suggests an involvement of E5 in the late stages of the viral life cycle [6].

Interestingly, BPV-1 E5 has also been found to be expressed in cutaneous fibropapillomas from water buffaloes [2]. This observation suggests that the oncoprotein plays a role in cutaneous fibropapillomas development independently of the infected species.

Typically E5 has been detected mostly within the cytoplasm of infected epithelial and fibroblastic cells, furthermore, some of the cells showed accentuated staining on one side of the nucleus, in a perinuclear region, consistent with the localization in the Golgi apparatus (GA) [30].

In E5 expressing fibropapillomas alterations in Connexin26 (Cx26) expression levels and in its intracellular localization have been demonstrated [31]. Interestingly, Cx26, a component of the skin connexons, colocalizes with E5 in a juxtanuclear region. This observation supports the hypothesis for a possible role of E5 in alteration of the Gap junctions intercellular communication mediated by $\mathrm{Cx} 26$ dysregulation. It is likely that the down-regulation of the gap junctions by E5 makes the transformed cells refractory to growth inhibitory signals from neighbouring cells [31]. It is worth noting that cutaneous fibropapillomas expressing E5 show hyperactivation of the PDGF $\beta R$ and its downstream transforming signals, suggesting a possible role of this cell signaling in lesion development [32].

BPV-E7 is expressed in the cytoplasm and nucleus of the spinous layers where vegetative BPV amplification is 
known to occur [6]. In bovine fibropapillomas E7 is localized in the basal layers of neoplastic epithelium where it colocalizes with E5. The co-expression of both proteins in the basal layer of fibropapillomas suggests that they may cooperate in controlling cell proliferation, and it is possible that E7 expression serves to modulate the actions of E5 on epithelial cells [6].

Remarkably, BPV-1 E7 expression in fibropapillomas correlates with the over-expression and the aberrant expression of $\beta 1$-integrin and PCNA in suprabasal epithelial cells. $\beta 1$-integrin is a member of the integrin family, which has a role in the regulation of keratinocytes proliferation and differentiation. The observation that the distribution of $\beta 1$-integrin within the fibropapilloma was reminiscent of the distribution of BPV-1 E7, indicates that E7 is implicated in the induction of vegetative DNA amplification in keratinocytes of the spinous layer [33].

Bovine fibropapillomas of the upper gastrointestinal tract The mucosal epithelium of the alimentary canal of cattle may be infected by BPV type 4 where it induces fibropapillomas (Figure 1B) [34]. Epithelial papillomas of the upper gastrointestinal (GI) tract can develop at every site from the mouth to the rumen. Histologically, they have the same features of benign fibropapillomas where the active viral replication results in inclusion bodies visible in the nuclei of infected cells $[35,36]$. Fibropapillomas regress in healthy immunocompetent animals due to an efficient cellmediated immune response. Cattle feeding on bracken fern develop upper alimentary canal cancers as a result of an interactions between the virus and the toxic and carcinogenic substances contained in bracken fern [8,37].

The transforming activities of BPV-4 is due to the viral genes E5 and E7. BPV-4 E5 encodes a small hydrophobic polypeptide similar in its length and amino acid composition to the BPV-1 E5 polypeptide (see above). BPV-4 E7 unlike BPV-1 E7 possesses the canonical Cys-X-X-Cys motif and the $\mathrm{pRb}$ interaction domain, that is crucial for its transforming ability [25]. Both proteins are expressed in virally induced alimentary canal tumours: E5 is mostly expressed in early development stages, on the contrary E7 is produced throughout all stages of the fibropapilloma, suggesting its pivotal role in the maintenance of the proliferative state of the epithelia and its necessary role in cell transformation [38].

BPV-4 E5 is expressed in fibropapillomas of the alimentary canal where it localizes in the basal layers [38]. Following viral infection of basal keratinocyte, E5 induces down-regulation of surface MHCI expression. This is an important observation for the notion that E5 has a role in the establishment of BPV infection in fibropapillomas of the alimentary canal. E5 may contribute to BPV escape from immunosurveillance through the prevention of viral antigen presentation to immune effector cells [39].
In alimentary carcinomas E7 was uniformly detected within the nuclei of basal and suprabasal cells and within the cytoplasm of differentiated spinous and granular cells, suggesting that this different intracellular localization might correspond to different functions [6].

The more extensive analysis of vaccination and immune response in relation to BPV-4 induced tumours was indicative for the possibility that E7 has an important role in cell-mediated immune responses leading to tumour rejection $[40,41]$. Experimental rejection of BPV4 papillomas has been achieved by immunizing cattle with the viral protein E7, and the regressing papillomas showed infiltrates of immune cells similar to those observed in naturally regressing tumours.

\section{Bovine urinary bladder tumours}

Tumours of the urinary bladder in cattle are of epithelial and mesenchymal origin (Figure 1C) [42,43] and commonly associated with chronic enzootic haematuria $(\mathrm{CEH})$, a syndrome caused by prolonged ingestion of bracken fern [8]. The involvement of BPV-1/2 in urinary bladder carcinogenesis is well recognized and the synergistic relationship between the virus and carcinogens from the fern has been experimentally reproduced [37].

The expression, localization and oncogenic activity of BPV E5 has been largely studied in BPV induced urinary bladder tumours. BPV E5 is expressed in the cytoplasm of both basal and suprabasal transformed urothelial cells. Expression of this oncoprotein is also seen in neoplastic blood vessels. E5 is found to be expressed intracytoplasmically with a typical juxtanuclear pattern suggesting Golgi localization [44-46]. This observation has been made on samples from different countries (Italy, Romania, Portugal, Brazil), confirming the specific localization of E5 protein in vivo $[44,47,48]$. The finding of E5 expression only in cancer samples but not in normal tissue suggests that the virus is involved in neoplastic processes and required for it [46]. In addition, intratumoral colocalization of E5 and E7 is noted, suggesting that cell transformation is triggered by these oncoproteins in a synergistic manner $[49,50]$.

The molecular mechanism by which E5 induces cell transformation lies in its binding to, and activation of the PDGF $\beta-r$, which is reflected by a potentiation of the mitogenic response [51]. Interestingly, Borzacchiello et al [52], have shown that in naturally occurring bovine urinary bladder tumours BPV E5 is able to physically interact with the PDGF $\beta-\mathrm{r}$ and that the receptor is more activated in cancer, confirming the role of the protein complex in cancer development. Moreover, the binding of the E5 and the PDGF $\beta-r$ induces the activation of different signal transduction pathways: the PDGF $\beta-r$ and PI3K physically interact such as PDGF $\beta-r$ and the Grb2Sos complex. PI3K-Akt and Grb2-Sos-Mek-Erk signals 
are all potentiated in cancer, but the levels of phosphorylated (activated) Erk and Mek proteins are not significantly overexpressed [53-55]. Cattle suffering from urinary bladder cancer harbour BPV DNA in blood cells where recently E5 expression has been demonstrated, supporting the hypothesis that blood may constitute a reservoir of the virus and suggesting a possible biological activity of BPV in the blood of these animals $[56,57]$.

It has been suggested that there is a possible direct or indirect role of E5 oncoprotein in the inhibition of intracellular communication in naturally occurring cancer. In fact, the expression of $\mathrm{Cx} 43$ was shown to be altered in different bovine urothelial neoplastic lesions expressing E5 oncoprotein. The expression of Cx43 was reduced in carcinoma in situ (CIS) samples, and was lost in neoplastic invasive urothelial cells suggesting a progressive loss of $\mathrm{Cx} 43$ during cancer development [58].

It is worth noting that the BPV-2 E5 oncoprotein has many biological characteristics similar to the viral HIV Negative Regulatory Factor (Nef) protein, which is involved in iron metabolism via the Ferritin heavy chain (HFE) [59]. The ferritin heavy chain is upregulated in E5 positive urothelial neoplasia [60]. The same samples express hepcidin (F Roperto, personal observations), an antimicrobial hormone known to be a key regulator in iron homeostasis. This observation suggests a possible involvement of E5 in iron homeostasis during the development of urothelial tumours.

During cell transformation, different cellular pathways are activated, among these the arachidonic acid metabolism seems to have an important role in tumour development [61]. E5 activates arachidonic acid metabolism independently of PDGF $\beta-r$ activation [62]. Interestingly, in spontaneously arising bovine urinary bladder carcinomas the cyclooxygenase enzymes are overexpressed, confirming a possible role of the E5 in the upregulation of arachidonic acid metabolism also in vivo [45].

Finally E5-expressing bovine urinary bladder tumours show activated calpain 3, suggesting a possible involvement of this protein in urothelial carcinogenesis [63]. It has been suggested that Calpain 3 plays a pro-apoptotic role in cancer development [64].

Interestingly, BPV-2 E5 has also been found to be expressed in urinary bladder tumours of water buffaloes [65]. This observation thus suggests that also in this case, the oncoprotein is involved in BPV-induced carcinogenesis independently of the infected species.

Finally BPV-2 E5 was found to be expressed and physically interacted with PDGF $\beta-\mathrm{r}$ in the uterine and chorionic epithelium of the placenta in pregnant cows suffering from naturally occurring papillomavirusassociated urothelial bladder tumours. It is possible that E5 action on PDGF $\beta-r$ is involved in organogenesis and neo-angiogenesis rather than in cell transformation during pregnancy [66].

\section{Equine sarcoid}

$\mathrm{BPV}$ is the only known case among PVs to cross infect other species. BPV-1/2 are able to infect equids inducing a common dermatological neoplasm called sarcoid. There are different clinical presentations of sarcoids (nodular, occult, fibroblastic, verrucous, malevolent and mixed, which is a combination of several of these types) (Figure 1D), but histologically they have all the same features: dermal proliferation of spindle-shaped fibroblasts features forming whorls and epidermal hyperplasia $[1,67]$.

In sarcoids the E5 oncogene is transcriptionally active and the protein is expressed in the neoplastic fibroblasts and overlying hyperplastic epidermis where the virus completes its life cycle producing virions particles [68-71]. The levels of E5 m-RNA are higher in the nodular type of sarcoids as compared with the other clinical types, although a recent report indicates that the highest levels of E5 DNA are found in the lesions of horses suffering from an aggressive form of disease i.e. multiple, rapidly growing tumours of various type [72,73]. These data provide all evidence of active BPV infection in sarcoids supporting the role of E5 in cell transformation. Interestingly, sequence analysis of the E5 open reading frame of sarcoidassociated BPV revealed several unique DNA sequence variants, some of which are associated with increased codon usage $[74,75]$. E5 sequence variations can influence the cellular location and function of the oncoprotein and are contributing factors to the pathogenesis of the disease. Sarcoid lesions co-express BPV E5 and E7 oncoproteins in neoplastic fibroblasts. E5 is localized in the cytoplasm in a juxtanuclear position, whereas E7 is found expressed in the cytoplasm and nuclei; the co-expression of E5 and E7, suggests that these molecules may co-operate also in sarcoid development. The role of E5 in naturally occurring sarcoids has been largely investigated: E5 is found in molecular complex with the phosphorylated PDGF $\beta-r$ (pPDGF $\beta-r$ ) in sarcoids and the high levels of pPDGF $\beta-r$ activate the PI3-K-Akt pathway and Grb2-Sos-Mek-Erk pathway (G Borzacchiello, personal observations) such as in bovine tumours [76]. It is therefore reasonable to assume that E5 can activate the same signal transduction pathways in bovine and equine species via the activation of the PDGF $\beta-r$.

E5 is also found transcriptionally active in blood cells from sarcoid affected horses, thus suggesting a possible contribution to virus spread. The BPV genomes and, more importantly, the expression of E5 in equine inflammatory skin conditions suggest that BPV is also involved in this type of disease [77].

Very little is known about the carcinogenic role of E7 in equine sarcoids, but it has recently been shown that 


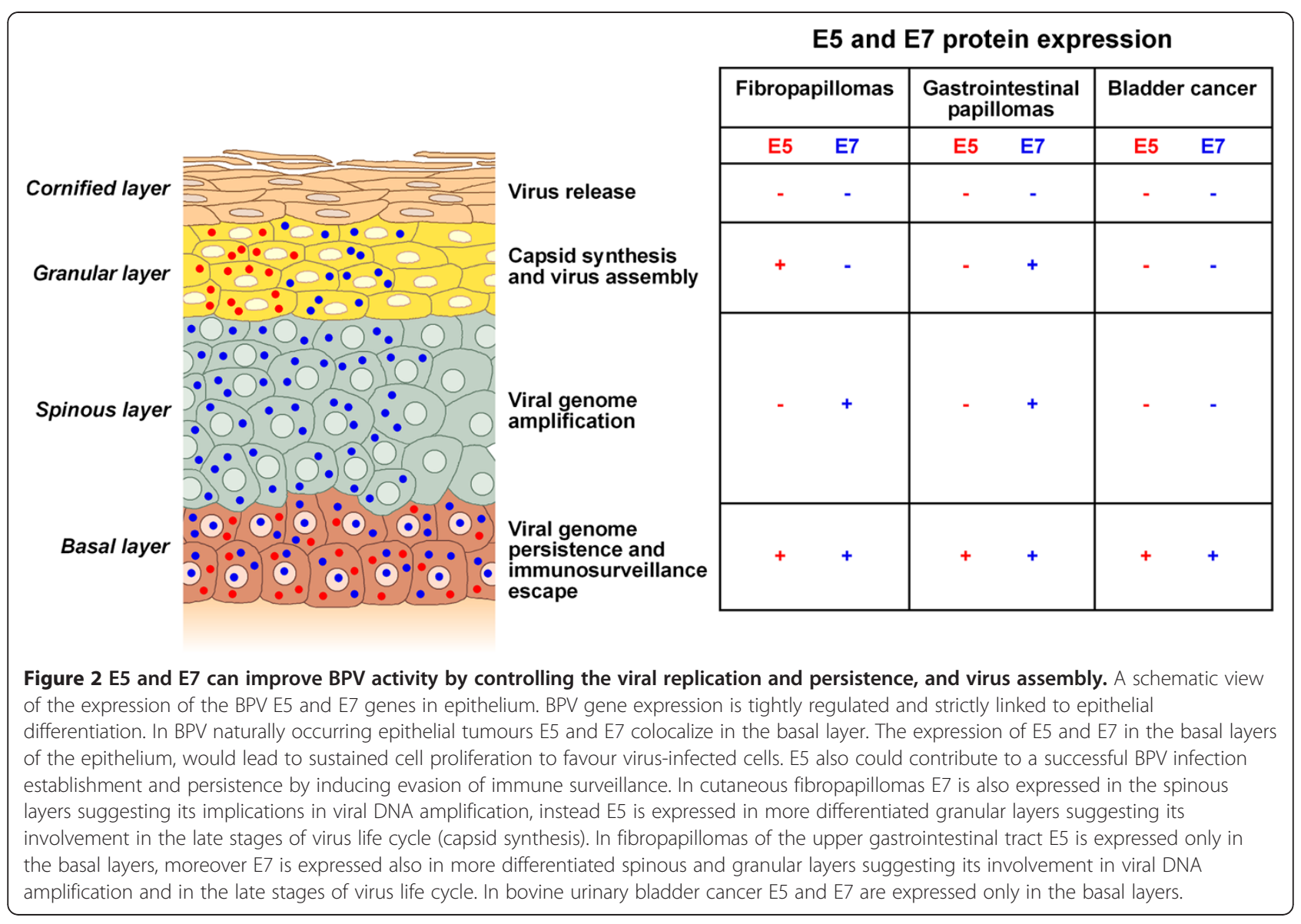

the interaction between the $600-\mathrm{kDa}$ retinoblastoma protein-associated factor (p600) and BPV E7, described in vitro in cultured cells, takes place also in vivo [78]. Furthermore, intracellular signals involved in p600 functional activity are not overexpressed, suggesting a different functional activity of p600 in naturally occurring carcinogenesis. It has also recently showed that E7 may contribute to upregulate the expression of MMP-1 as suggested by a previous in vivo study $[79,80]$. These findings suggest that E7 may contribute to sarcoid cell invasion process through upregulation of MMP.

\section{Conclusions}

PVs induced cell transformation is paradigmatically based on the different contribution of the three viral oncogenes E5, E6, and E7 affecting multiple cellular pathways.

Most of the studies about PV induced oncogenesis and the role of the viral oncogenes have firstly been recognized in the BPV model system and later validated for HPV.

The expression and functions of BPV E5 and E7 oncoproteins have been largely investigated in naturally

Bovine fibropapillomas
\begin{tabular}{|l|}
\hline E5 biological function \\
PDGF $\beta$ R activation \\
pAKT up-regulation \\
Cx26 aberrant expression \\
MHCI down-regulation \\
E7 biological function \\
$\beta 1$ integrin overexpression \\
PCNA aberrant expression \\
\hline
\end{tabular}

Bovine bladder cancer

E5 biological function PDGF $\beta$ R activation

PI3K pathway up-regulation

Ras-MAPK pathway up-regulation

Cx43 down-regulation

Iron omeostasis

Ciclooxygenase overexpression

Calpain3 activation

\begin{tabular}{l} 
Equine sarcoid \\
\begin{tabular}{|l|}
\hline E5 biological function \\
PDGF $\beta$ R activation \\
PI3K pathway up-regulation \\
E7 biological function \\
P600 activation \\
MMP1 activation \\
\hline
\end{tabular} \\
\hline
\end{tabular}

Figure 3 Schematic representation of cellular events mediated by BPV E5 and E7 responsible of cell transformation in naturally occurring tumours. 
occurring tumours (Figure 2) with E5 being the major oncogene. Recent studies about the functions of E5 in vivo have on the one hand confirmed in vitro studies, on the other hand have discovered new aspects.

The role of BPV E7 in cellular transformation has been less investigated. To date, in vivo studies suggest a synergism between E5 and E7: they cooperate in the BPV infection establishment and persistence as well as in controlling cell proliferation and transformation processes (Figure 3), suggesting that "two are better than one" in naturally occurrying carcinogenesis.

Finally, in vivo studies about the expression and functions of BPV oncoproteins in experimentally PV-induced tumours are still relevant to better understand the oncogenic potential of the virus and to gain new insights into the interplay between PVs oncogenes.

\section{Competing interests}

The authors declared that they have no competing interests.

None of the authors of this paper has financial or personal relationship with other people or organizations that could innappropriately influence or bias the content of the paper.

\section{Authors' contributions}

All authors have contributed equally to the paper. All authors read and approved the final manuscript.

\section{Acknowledgments}

The authors are grateful to Prof. Annamaria Lamarra at Centro Linguistico di Ateneo, University of Naples Federico II, for her editing of the manuscript. This work was supported by a grant from Ministero Istruzione Università e Ricerca Scientifica (MIUR): PRIN 2008: Meccanismi di cancerogenesi in neoplasie animali indotte da papillomavirus bovino- prot. № 2008LTY389.

Received: 5 October 2012 Accepted: 7 January 2013

Published: 9 January 2013

\section{Reference}

1. Nasir $L$, Campo MS: Bovine papillomaviruses: their role in the aetiology of cutaneous tumours of bovids and equids. Vet Dermatol 2008, 19(5):243-254.

2. Silvestre O, Borzacchiello G, Nava D, lovane G, Russo V, Vecchio D, D'Ausilio F, Gault EA, Campo MS, Paciello O: Bovine papillomavirus type 1 DNA and E5 oncoprotein expression in water buffalo fibropapillomas. Vet Pathol 2009, 46(4):636-641.

3. Tomita Y, Literak I, Ogawa T, Jin Z, Shirasawa H: Complete genomes and phylogenetic positions of bovine papillomavirus type 8 and a variant type from a european bison. Virus Genes 2007, 35(2):243-249.

4. Campo MS: Papillomavirus research: from natural history to vaccines and beyond. Wymondham: Caister Academic; 2006:xi-423 p.

5. Venuti A, Paolini F, Nasir L, Corteggio A, Roperto S, Campo MS, Borzacchiello G: Papillomavirus E5: the smallest oncoprotein with many functions. Mol Cancer 2011, 10(1):140.

6. Bohl J, Hull B, Vande Pol SB: Cooperative transformation and coexpression of bovine papillomavirus type 1 E5 and E7 proteins. J Virol 2001, 75(1):513-521.

7. Borzacchiello G, Roperto F, Nasir L, Campo MS: Human papillomavirus research: do we still need animal models? Int J Cancer 2009, 125(3):739-740

8. Borzacchiello G, Roperto F: Bovine papillomaviruses, papillomas and cancer in cattle. Vet Res 2008, 39(5):45.

9. Jackson ME, Pennie WD, McCaffery RE, Smith KT, Grindlay GJ, Campo MS: The B subgroup bovine papillomaviruses lack an identifiable E6 open reading frame. Mol Carcinog 1991, 4(5):382-387.

10. Tong X, Howley PM: The bovine papillomavirus E6 oncoprotein interacts with paxillin and disrupts the actin cytoskeleton. Proc Natl Acad Sci USA 1997, 94(9):4412-4417.
11. Das K, Bohl J, Vande Pol SB: Identification of a second transforming function in bovine papillomavirus type $1 \mathrm{E} 6$ and the role of $\mathrm{E} 6$ interactions with paxillin, E6BP, and E6AP. J Virol 2000, 74(2):812-816.

12. Burkhardt A, DiMaio D, Schlegel R: Genetic and biochemical definition of the bovine papillomavirus E5 transforming protein. EMBO J 1987, 6(8):2381-2385.

13. DiMaio $D$, Guralski D, Schiller JT: Translation of open reading frame $E 5$ of bovine papillomavirus is required for its transforming activity. Proc Natl Acad Sci USA 1986, 83(6):1797-1801.

14. Schiller JT, Vass WC, Vousden KH, Lowy DR: E5 Open reading frame of bovine papillomavirus type 1 encodes a transforming gene. J Virol 1986, 57(1):1-6.

15. Schlegel R, Wade-Glass M, Rabson MS, Yang YC: The E5 transforming gene of bovine papillomavirus encodes a small, hydrophobic polypeptide. Science 1986, 233(4762):464-467.

16. Xu YF, Meyer AN, Webster MK, Lee BA, Donoghue DJ: The v-sis protein retains biological activity as a type II membrane protein when anchored by various signal-anchor domains, including the hydrophobic domain of the bovine papilloma virus E5 oncoprotein. J Cell Biol 1993, 123(3):549-560.

17. Horwitz BH, Burkhardt AL, Schlegel R, DiMaio D: 44-Amino-acid E5 transforming protein of bovine papillomavirus requires a hydrophobic core and specific carboxyl-terminal amino acids. Mol Cell Biol 1988, 8(10):4071-4078.

18. Sparkowski J, Anders J, Schlegel R: E5 Oncoprotein retained in the endoplasmic reticulum/cis golgi still induces PDGF receptor autophosphorylation but does not transform cells. EMBO J 1995, 14(13):3055-3063.

19. Kulke R, DiMaio D: Biological properties of the deer papillomavirus E5 gene in mouse $\mathrm{C} 127$ cells: growth transformation, induction of DNA synthesis, and activation of the platelet-derived growth factor receptor. $J$ Virol 1991, 65(9):4943-4949.

20. Sarver N, Rabson MS, Yang YC, Byrne JC, Howley PM: Localization and analysis of bovine papillomavirus type 1 transforming functions. J Virol 1984, 52(2):377-388.

21. Munger K, Werness BA, Dyson N, Phelps WC, Harlow E, Howley PM: Complex formation of human papillomavirus E7 proteins with the retinoblastoma tumor suppressor gene product. EMBO J 1989, 8(13):4099-4105.

22. DeMasi J, Chao MC, Kumar AS, Howley PM: Bovine papillomavirus E7 oncoprotein inhibits anoikis. J Virol 2007, 81(17):9419-9425.

23. Liu Y, Hong Y, Androphy EJ, Chen JJ: Rb-independent induction of apoptosis by bovine papillomavirus type $1 \mathrm{E} 7$ in response to tumor necrosis factor alpha. J Biol Chem 2000, 275(40):30894-30900.

24. Fan X, Liu Y, Chen JJ: Activation of c-Myc contributes to bovine papillomavirus type 1 E7-induced cell proliferation. J Biol Chem 2003, 278(44):43163-43168.

25. Pennie WD, Grindlay GJ, Cairney M, Campo MS: Analysis of the transforming functions of bovine papillomavirus type 4. Virology 1993 , 193(2):614-620.

26. Jaggar RT, Pennie WD, Smith KT, Jackson ME, Campo MS: Cooperation between bovine papillomavirus type 4 and ras in the morphological transformation of primary bovine fibroblasts. J Gen Virol 1990, 71(Pt 12):3041-3046.

27. Hatama S, Ishihara R, Ueda Y, Kanno T, Uchida I: Detection of a novel bovine papillomavirus type 11 (BPV-11) using xipapillomavirus consensus polymerase chain reaction primers. Arch Virol 2011, 156(7):1281-1285.

28. Lunardi M, Alfieri AA, Otonel RA, de Alcantara BK, Rodrigues WB, de Miranda $A B$, Alfieri AF: Genetic characterization of a novel bovine papillomavirus member of the deltapapillomavirus genus. Vet Microbiol 2012, in press.

29. Campo MS: Infection by bovine papillomavirus and prospects for vaccination. Trends Microbiol 1995, 3(3):92-97.

30. Burnett S, Jareborg N, DiMaio D: Localization of bovine papillomavirus type $1 \mathrm{E} 5$ protein to transformed basal keratinocytes and permissive differentiated cells in fibropapilloma tissue. Proc Natl Acad Sci USA 1992, 89(12):5665-5669.

31. Silva MA, Altamura G, Corteggio A, Roperto F, Bocaneti F, Velescu E, Freitas AC, Carvalho CC, Cavalcanti KP, Borzacchiello G: Expression of connexin 26 and bovine papillomavirus E5 in cutaneous fibropapillomas of cattle. Vet $J$ 2012, in press.

32. Bocaneti F, Altamura G, Corteggio A, Martano M, Roperto F, Velescu E, Borzacchiello G: Expression of platelet derived growth factor beta 
receptor, its activation and downstream signals in bovine cutaneous fibropapillomas. Res Vet Sci 2012, in press.

33. Cooper B, Brimer N, Stoler M, Vande Pol SB: Suprabasal overexpression of beta-1 integrin is induced by bovine papillomavirus type 1. Virology 2006, 355(1):102-114.

34. Saveria Campo M, Moar MH, Jarrett WF, Laird HM: A new papillomavirus associated with alimentary cancer in cattle. Nature 1980, 286(5769):180-182.

35. Borzacchiello G, Ambrosio V, Roperto S, Poggiali F, Tsirimonakis E, Venuti A Campo MS, Roperto F: Bovine papillomavirus type 4 in oesophageal papillomas of cattle from the south of italy. J Comp Pathol 2003 128(2-3):203-206.

36. Hamada M, Oyamada T, Yoshikawa H, Yoshikawa T: Morphological studies of esophageal papilloma naturally occurring in cattle. Nihon Juigaku Zasshi 1989, 51(2):345-351.

37. Campo MS, Jarrett WF, Barron R, O'Neil BW, Smith KT: Association of bovine papillomavirus type 2 and bracken fern with bladder cancer in cattle. Cancer Res 1992, 52(24):6898-6904.

38. Anderson RA, Scobie L, O'Neil BW, Grindlay GJ, Campo MS: Viral proteins of bovine papillomavirus type 4 during the development of alimentary canal tumours. Vet J 1997, 154(1):69-78

39. Araibi EH, Marchetti B, Ashrafi GH, Campo MS: Downregulation of major histocompatibility complex class I in bovine papillomas. J Gen Virol 2004, 85(Pt 10):2809-2814

40. Campo MS, Grindlay GJ, O'Neil BW, Chandrachud LM, McGarvie GM, Jarrett WF: Prophylactic and therapeutic vaccination against a mucosal papillomavirus. J Gen Virol 1993, 74(Pt 6):945-953.

41. Knowles G, O'Neil BW, Campo MS: Phenotypical characterization of lymphocytes infiltrating regressing papillomas. J Virol 1996, 70(12):8451-8458.

42. Borzacchiello G, Ambrosio V, Galati P, Poggiali F, Venuti A, Roperto F: The pagetoid variant of urothelial carcinoma in situ of urinary bladder in a cow. Vet Pathol 2001, 38(1):113-116.

43. Borzacchiello G, Ambrosio V, Leonardi L, Fruci R, Galati P, Roperto F: Rare tumours in domestic animals: a lipid cell variant of urothelial carcinoma of the urinary bladder in a cow and a case of vesical carcinosarcoma in a dog. Vet Res Commun 2004, 28(Suppl 1):273-274

44. Balcos LG, Borzacchiello G, Russo V, Popescu O, Roperto S, Roperto F: Association of bovine papillomavirus type- 2 and urinary bladder tumours in cattle from romania. Res Vet Sci 2008, 85(1):145-148.

45. Borzacchiello G, Ambrosio V, Galati P, Perillo A, Roperto F: Cyclooxygenase1 and -2 expression in urothelial carcinomas of the urinary bladder in cows. Vet Pathol 2003, 40(4):455-459.

46. Roperto S, Borzacchiello G, Brun R, Leonardi L, Maiolino P, Martano M, Paciello O, Papparella S, Restucci B, Russo V, Salvatore G, Urraro C, Roperto F: A review of bovine urothelial tumours and tumour-like lesions of the urinary bladder. J Comp Pathol 2010, 142(2-3):95-108.

47. Borzacchiello G, lovane G, Marcante ML, Poggiali F, Roperto F, Roperto S, Venuti A: Presence of bovine papillomavirus type 2 DNA and expression of the viral oncoprotein E5 in naturally occurring urinary bladder tumours in cows. J Gen Virol 2003, 84(Pt 11):2921-2926.

48. Resendes AR, Roperto S, Trapani F, Urraro C, Rodrigues A, Roperto F, Borzacchiello G: Association of bovine papillomavirus type 2 (BPV-2) and urinary bladder tumours in cattle from the azores archipelago. Res Vet SCi 2011, 90(3):526-529.

49. Borzacchiello G, Russo V, Spoleto C, Roperto S, Balcos L, Rizzo C, Venuti A, Roperto F: Bovine papillomavirus type-2 DNA and expression of E5 and E7 oncoproteins in vascular tumours of the urinary bladder in cattle. Cancer Lett 2007, 250(1):82-91.

50. Borzacchiello G, Resendes AR, Roperto S, Roperto F: Co-expression of bovine papillomavirus E5 and E7 oncoproteins in naturally occurring carcinomas of the urinary bladder in cattle. J Comp Pathol 2009, 141(1):84-88.

51. DiMaio D, Mattoon D: Mechanisms of cell transformation by papillomavirus E5 proteins. Oncogene 2001, 20(54):7866-7873.

52. Borzacchiello G, Russo V, Gentile F, Roperto F, Venuti A, Nitsch L, Campo MS, Roperto S: Bovine papillomavirus E5 oncoprotein binds to the activated form of the platelet-derived growth factor beta receptor in naturally occurring bovine urinary bladder tumours. Oncogene 2006, 25(8):1251-1260.

53. Corteggio A, Urraro C, Roperto S, Roperto F, Borzacchiello G: Phosphatidylinositol-3-kinase-AKT pathway, phospho-JUN and phospho-JNK expression in spontaneously arising bovine urinary bladder tumours. J Comp Pathol 2010, 143(2-3):173-178.
54. Corteggio A, Di Geronimo O, Roperto S, Roperto F, Borzacchiello G: Activated platelet-derived growth factor beta receptor and Ras-mitogenactivated protein kinase pathway in natural bovine urinary bladder carcinomas. Vet J 2011, 191(3):393-395.

55. Petti LM, Ricciardi EC, Page HJ, Porter KA: Transforming signals resulting from sustained activation of the PDGFbeta receptor in mortal human fibroblasts. J Cell Sci 2008, 121(Pt 8):1172-1182.

56. Campo MS, Jarrett WF, O'Neil W, Barron RJ: Latent papillomavirus infection in cattle. Res Vet Sci 1994, 56(2):151-157.

57. Roperto S, Brun R, Paolini F, Urraro C, Russo V, Borzacchiello G, Pagnini U, Raso C, Rizzo C, Roperto F, Venuti A: Detection of bovine papillomavirus type 2 in the peripheral blood of cattle with urinary bladder tumours: possible biological role. J Gen Virol 2008, 89(Pt 12):3027-3033.

58. Corteggio A, Florio J, Roperto F, Borzacchiello G: Expression of gap junction protein connexin 43 in bovine urinary bladder tumours. J Comp Pathol 2011, 144(1):86-90.

59. Tsirimonaki E, Ullah R, Marchetti B, Ashrafi GH, McGarry L, Ozanne B, Campo MS: Similarities and differences between the $\mathrm{E} 5$ oncoproteins of bovine papillomaviruses type 1 and type 4: cytoskeleton, motility and invasiveness in E5-transformed bovine and mouse cells. Virus Res 2006, 115(2):158-168.

60. Roperto S, Borzacchiello G, Brun R, Costanzo F, Faniello MC, Raso C, Rosati A, Russo V, Leonardi L, Saracino D, Turco MC, Urraro C, Roperto F: Ferritin heavy chain $(\mathrm{FHC})$ is up-regulated in papillomavirus-associated urothelial tumours of the urinary bladder in cattle. J Comp Pathol 2010, 142(1):9-18

61. Marks F, Furstenberger G, Muller-Decker K: Metabolic targets of cancer chemoprevention: interruption of tumor development by inhibitors of arachidonic acid metabolism. Recent Results Cancer Res 1999, 151:45-67.

62. Vali U, Kilk A, Ustav M: Bovine papillomavirus oncoprotein E5 affects the arachidonic acid metabolism in cells. Int J Biochem Cell Biol 2001, 33(3):227-235

63. Roperto S, De Tullio R, Raso C, Stifanese R, Russo V, Gaspari M, Borzacchiello G, Averna M, Paciello O, Cuda G, Roperto F: Calpain3 Is expressed in a proteolitically active form in papillomavirus-associated urothelial tumors of the urinary bladder in cattle. PLoS One 2010, 5(4):e10299.

64. Moretti D, Del Bello B, Cosci E, Biagioli M, Miracco C, Maellaro E: Novel variants of muscle calpain 3 identified in human melanoma cells: cisplatin-induced changes in vitro and differential expression in melanocytic lesions. Carcinogenesis 2009, 30(6):960-967.

65. Roperto S, Russo V, Ozkul A, Sepici-Dincel A, Maiolino P, Borzacchiello G, Marcus I, Esposito I, Riccardi MG, Roperto F: Bovine papillomavirus type 2 infects the urinary bladder of water buffalo (bubalus bubalis) and plays a crucial role in the bubaline urothelial carcinogenesis. J Gen Viro/ 2012, in press.

66. Roperto S, Borzacchiello G, Esposito I, Riccardi M, Urraro C, Luca R, Corteggio A, Tate R, Cermola M, Paciello O, Roperto F: Productive infection of bovine papillomavirus type 2 in the placenta of pregnant cows affected with urinary bladder tumors. PLoS One 2012, 7(3):e33569.

67. Pascoe RR, Knottenbelt DC: Manual of equine dermatology. New York: W.B. Saunders; 1999:x-290 p.

68. Carr EA, Theon AP, Madewell BR, Griffey SM, Hitchcock ME: Bovine papillomavirus DNA in neoplastic and nonneoplastic tissues obtained from horses with and without sarcoids in the western united states. Am J Vet Res 2001, 62(5):741-744.

69. Borzacchiello G, Russo V, Della Salda L, Roperto S, Roperto F: Expression of platelet-derived growth factor-beta receptor and bovine papillomavirus E5 and E7 oncoproteins in equine sarcoid. J Comp Pathol 2008, 139(4):231-237.

70. Brandt S, Tober R, Corteggio A, Burger S, Sabitzer S, Walter I, Kainzbauer C, Steinborn R, Nasir L, Borzacchiello G: BPV-1 infection is not confined to the dermis but also involves the epidermis of equine sarcoids. Vet Microbio/ 2011, 150(1-2):35-40

71. Nasir L, Reid SW: Bovine papillomaviral gene expression in equine sarcoid tumours. Virus Res 1999, 61(2):171-175.

72. Bogaert L, Van Poucke M, De Baere C, Dewulf J, Peelman L, Ducatelle R, Gasthuys F, Martens A: Bovine papillomavirus load and mRNA expression, cell proliferation and p53 expression in four clinical types of equine sarcoid. J Gen Virol 2007, 88(Pt 8):2155-2161.

73. Haralambus R, Burgstaller J, Klukowska-Rotzler J, Steinborn R, Buchinger S, Gerber V, Brandt S: Intralesional bovine papillomavirus DNA loads reflect severity of equine sarcoid disease. Equine Vet J 2010, 42(4):327-331. 
74. Chambers G, Ellsmore VA, O'Brien PM, Reid SW, Love S, Campo MS, Nasir L: Sequence variants of bovine papillomavirus E5 detected in equine sarcoids. Virus Res 2003, 96(1-2):141-145.

75. Reid SW, Smith KT, Jarrett WF: Detection, cloning and characterisation of papillomaviral DNA present in sarcoid tumours of equus asinus. Vet Rec 1994, 135(18):430-432.

76. Borzacchiello G, Mogavero S, De Vita G, Roperto S, Della Salda L, Roperto F: Activated platelet-derived growth factor beta receptor expression, PI3KAKT pathway molecular analysis, and transforming signals in equine sarcoids. Vet Pathol 2009, 46(4):589-597.

77. Yuan Z, Philbey AW, Gault EA, Campo MS, Nasir L: Detection of bovine papillomavirus type 1 genomes and viral gene expression in equine inflammatory skin conditions. Virus Res 2007, 124(1-2):245-249.

78. Corteggio A, Di Geronimo O, Roperto S, Roperto F, Borzacchiello G: Bovine papillomavirus E7 oncoprotein binds to p600 in naturally occurring equine sarcoids. J Gen Virol 2011, 92(Pt 2):378-382.

79. Yuan Z, Gault EA, Campo MS, Nasir L: Upregulation of equine matrix metalloproteinase 1 by bovine papillomavirus type 1 is through the transcription factor activator protein-1. J Gen Virol 2011, 92(Pt 11):2608-2619.

80. Yuan Z, Gobeil PA, Campo MS, Nasir L: Equine sarcoid fibroblasts overexpress matrix metalloproteinases and are invasive. Virology 2010, 396(1):143-151.

doi:10.1186/1750-9378-8-1

Cite this article as: Corteggio et al:: Bovine papillomavirus E5 and E7 oncoproteins in naturally occurring tumors: are two better than one?. Infectious Agents and Cancer 2013 8:1.

\section{Submit your next manuscript to BioMed Central and take full advantage of:}

- Convenient online submission

- Thorough peer review

- No space constraints or color figure charges

- Immediate publication on acceptance

- Inclusion in PubMed, CAS, Scopus and Google Scholar

- Research which is freely available for redistribution 